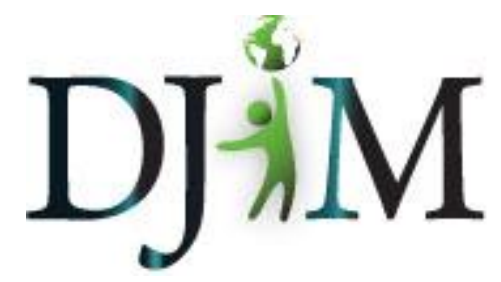

Volume 8 - Spring 2012 djim.management.dal.ca

\title{
Qualitative Evidence, Knowledge Translation, and Policymaking, with Reference to Health Technology Assessment
}

\begin{abstract}
Although efforts to draw qualitative evidence into health-related policymaking and health technology assessment (HTA) processes have increased in recent years, the range of sources consulted are still limited and the theoretical foundations for consulting them are underdeveloped. This essay builds on such recent scholarship by opening up conventional models of knowledge translation to the possibilities of qualitative evidence and by demonstrating the utility of this wider range of qualitative evidence - signally that of humanities scholarship - in health-related policymaking. The essay will use themes of pain and narrativity to illustrate both the particular complexity of policymaking in HTA - whereby social, ethical, and moral variables are at play and the mitigating effect humanities scholarship, at its best, might have on this fraught process.
\end{abstract}

\footnotetext{
About the Author(s): Jordan K. Penney was born and raised in Atlantic Canada and educated in England where, as a Graduate Fellow with the Centre for Renaissance and Early Modern Studies at the University of York, he obtained a PhD in History. He also holds a BA and an MA in that discipline from Dalhousie University. He is currently an MPA student in Dalhousie's School of Public Administration, and would like to acknowledge Professor Dominika Wranik for putting his mind to the subject of health technology assessment policy in the first place.
} 


\section{Introduction}

Although humanities research is routinely deployed as an instrument for shaping and delivering social policies, its potential in the field of health care policy is largely unrealized. Conventional models for knowledge translation and policy design and implementation in health care are oriented towards the use of quantitative evidence derived from clinical and economic data. Where such guidelines also incorporate societal or ethical values as criteria for decisionmaking, their full implications are not typically perceived. The broader implications of such qualitative evidence, particularly that of the humanities, will be explored, and a case will be made for why this evidence has a place in health-related policy procedures. This essay will suggest that qualitative humanities research could have a stronger appeal and be a more compelling force as the agenda-setting, formulation, and policymaking processes are carried out in health-related fields. By way of grounding these arguments in policy settings, specific references will be made to policy decisions concerning health technology assessments (HTA) - an area that is already showing the potential of qualitative evidence. These policy settings consist of clinicians, health economists, policy advisers/researchers, decision-makers, and non-specialist participants.

This essay will consist of three parts. The first and second parts will clarify the phrases, categories, and theories that will appear throughout. The most central of these is knowledge translation, which is recognized in health care policy as an instrument for bringing various participants together and facilitating the communication of technical knowledge. Its proponents claim interdisciplinarity as its strength, but its application in practice is nevertheless quite narrow. In the second part of this essay, an argument will be made for qualitative and humanities research to play a might significantly useful necessary role in policy deliberations. This part of the essay will also describe how qualitative evidence either is used by actors in fragmented ways, or is not used by actors, although its presence could be helpful. The third and final part of this essay will consist of examples of two themes - pain and narrative - that are examined at length by humanities scholars and are entirely relevant to health-related policy.

\section{Definitional Clarity}

Before proceeding further, it is necessary to define the relevant concepts. The humanities as a discipline, or a collection of closely related disciplines, is understood here as the speculative and critical work characteristic of philosophy, literature, history, and cultural studies. Its philosophical basis is grounded in the categories suggested by Giacomini et al. (2010), of which there are three: ontological - "[l]ooks at social meaning, interaction, contexts," 
epistemological -“[p]ursues inductive interpretation, description," and ideological - "[o]ften empowerment -- voice, translation, exposé" (p. 5). To these three one may add a fourth, phenomenological - the philosophy of the 'self' and how it shapes (and is shaped by) other self-determining selves.

In health care literature, qualitative evidence is usually associated with data generated by the social sciences - namely political science, sociology, and anthropology - where methodologies are not strictly quantitative in their respective orientations (Landry, Amara, \& Lamari, 2001). Even if the term "evidence" itself is ambiguous, humanities research and the conclusions it advances may be understood as properly qualitative in a broad sense as "observation, fact or organized body of information, offered to support or justify inferences or beliefs in the demonstration of some proposition or matter at issue" (Upshur, VanDenKerhof, \& Goel, 2001, p. 93). Since the use of such evidence or data is here considered part of the dynamic processes associated with policy decisions, its potential role in health care knowledge translation is important (Graham et al., 2006; Howlett, Ramesh, \& Perl, 1995). There are a variety of ways to characterize knowledge translation. Here the definition provided by the Canadian Institutes of Health Research will be preferred: "exchange, synthesis and ethicallysound application of knowledge--within a complex system of interactions among researchers and users" (Tetro, 2007, p. 1).

Where knowledge translation, so defined, has appeared as an instrument for policymaking, it has recognized the challenges presented by communication between practitioners of diverse disciplines. These participants typically include public policymakers, elected officials, political advisers, and civil servants (Lavis, 2006, p. 37). Where health care is concerned, third-party participants might also be included. In this case, third-party participants consist of individuals directly affected by health care policy as well as researchers committed to understanding these experiences in terms conventional to humanities scholarship: as discursive, contextual, symbolic, and narrative-based. These contributions are qualitative, and knowledge translation in principle offers a model for either bringing them into the decision-making processes or enhancing the place they currently occupy in such processes.

\section{Knowledge Translation: Interdisciplinary or Not?}

Based on the brief sketch above, it would seem that the core of knowledge translation, with its diversity of participants and targets, is interdisciplinary. This fact is evident in the literature on knowledge translation theory. Davis (2006) describes knowledge translation as "interdisciplinary in the extreme" (p. 5). In this literature, there is an abundance of bridgerelated metaphors as well as more conventional pleas for community-building (Ho, Chockalingham, Best, \& Walsh, 2003). Knowledge translation itself might even be synonymous with "interprofessional collaboration" (Zwarenstein \& Reeves, 2006, p. 46). In the field of health care, whether interventions are technological, pharmacological, or in any general way policy-related, the process by which they have been initiated will be characterized in 
similar language: "successful healthcare management" is "measured by the objectives of patients, physicians, and other healthcare providers, as well as by societal expectations" (Bootman, Townsend, \& McGhan, 2005, p. 3). However, certainty of these objectives cannot be easily empirically derived. The language of measurement itself is rather inappropriate, and this is especially true with respect to subjective patient experiences.

Although qualitative and humanities research might shed light on subjective experiences, societal expectations, humanistic benefits, personal dignity, and other concepts pregnant with untapped meaning, this research is often not consulted. There is precedence in the literature of HTA going as far back as 1976 for declaring the importance of a diverse range of qualitative input under the broad rubric of "social impacts" (Office of Technology Assessment, 1976, p. vii). In principle this has been parsed variously as societal, ethical, and moral inputs, and as the foregoing analyses of some models of knowledge translation will show, the space allowed for humanities research into these variables is small. A recent study (Lehoux, Tailliez, Denis \& Hivon, 2004) of how the variables involved in HTA have been balanced in practice between 1995 and 2001 affirms that "HTA should examine not only the efficacy, safety, and costs of health technology, but also the ethical, social, and legal issues." The study concludes that "ethical and social issues were not frequently addressed" (Lehoux, Tailliez, Denis \& Hivon, 2004 , p. 329). Whereas costs and effectiveness were most frequently addressed in HTA reports, ethical and social issues were consulted in only $17 \%$ of them (Lehoux, Tailliez, Denis \& Hivon, 2004). This reflects the outlook of other observers who lament that HTA is typically reduced to cost-effectiveness (Hughes, 2009), as well as that of Giacomini and her collaborators, who attest that it is "atypical" for policymakers to consult the humanities (Giacomini, Hurley, Gold, Smith \& Abelson, 2001, p. 16).

At the same, many policymakers, health care professions, health economists, and other scholars recognize the need for wider common ground in properly carrying out knowledge translation and responsible assessments. Some of these needs and the facilitating role that humanities research might assume in helping to establish common ground are mapped out below. First, the incomplete and inconsistent roles assigned to qualitative evidence in the literature are described. Second, the importance of certain qualitative forms of data in healthrelated fields is examined. Third, models for integrating explicitly qualitative data into healthrelated policymaking are identified. Finally, the mixed efforts of humanities scholars themselves, who have both helped and hindered the reception of their own work, are situated within the field of concern.

Despite exhortations to integrate diverse evidence into health care practices and policymaking, the disciplinary range is limited. Upshur et al. (2001) have observed a need for a synthesis of proof that legitimizes qualitative evidence in health care policy, and recognize that the field encompasses humanities as well as social sciences research. However, when they indicate that the qualitative contributions of the social sciences have received scant attention relative to the quantitative contributions, they fail to add that humanities contributions have received even 
less attention. This is puzzling because were the provisions of their inclusive model elaborated in full, it would legitimize qualitative evidence and humanities research as part of knowledge translation. Their approach outlines four distinct types of evidence: qualitative-personal, qualitative-general, quantitative-general, and quantitative-personal (Upshur et al, 2011, p. 91). The first of these four accommodates the sort of qualitative information produced by the humanities, and as such it is the category of concern here. It is defined thus:

Evidence is primarily narrative in nature, emerges from a historical context and is concerned with particular individuals. It involves the varying beliefs, attitudes, preferences, and 'epistemological frames' brought forth by both health-care provider and patient. (Upshur et al., 2011, p. 94)

Its philosophical underpinnings are hermeneutical, ethnographical, sociological, and anthropological (Upshur et al., 2011, p. 94). Hermeneutics is a technical extension of literary interpretation and it will not feature in this essay. However, its appearance in the model has the effect of allowing for a wide range of intervention under the qualitative-personal rubric in the formula. It creates a space for inclusion of evidence derived from the personal and the particular, and more precisely, the symbolic, discursive, context-specific, and narrative-based.

The possibilities of these interventions have not gone unnoticed. There is a field of scholarship devoted entirely to bringing together the qualitative and quantitative in medicine and health care: the medical humanities. An academic journal now bears that name (Evans \& Greaves, 2010). In the words of Jane Harden (2000), 'narrative knowing', an idea that features widely in this scholarship, can be told through:

case history (clinical) and life story (theory-building). The life story of the individual as a patient represents him or her to the nurse and is in turn responded to through appropriate care. Alternatively, the life story of the individual as research participant represents him or her to the researcher and is in turn responded to through appropriate interpretation. (p. 506)

Consequently there is a tradition in medical training that attempts to come to grips with narrativity, patient-centred care, and the value of the patient's point of view (Shapiro, 2011, p. $68)$.

In the field of health economics there have been responses to the challenges of translating cost-effective or cost-utility evidence for policymakers. Although the role that economic evidence takes in the allocation of public resources for health care is fraught with its own respective difficulties, there is an element related to this objective that is pertinent to the present task. This element is patient participation in the formal process whereby health care professionals, health economists, and other partners carry out evaluations and advise policy decision-makers on technological (including pharmaceutical) interventions (Wranik, 2008). 
Patient representation in this process has been in part a feature of the drive towards knowledge translation in general and improved transparency in the decision-making process in particular. The range of those invited to participate in policy decisions encompasses stakeholders - including patients and service users - as well as the broader citizenry (Bombard, Abelson, Simeonov, \& Gauvin, 2011). There are also basic common law requirements that stipulate conditions of procedural fairness, which are arguably contravened by closed-door decision-making (Attaran, 2009, p. 12-13). Whereas the Canadian Common Drug Review, for example, lacks mechanisms for patient and general public involvement (Attaran, 2009, p. 13), the Pan-Canadian Oncology Drug Review's new process (as of 2011) "will include input from patient groups, drug manufacturers, clinicians, and government," along with health economists ("Pan-Canadian Cancer Drug Review," 2011, para. 2). However, if health economists already have difficulty translating their findings for policymakers who are rarely equipped to properly digest the technical knowledge that accompanies cost-effective and cost-utility analyses (Wranik, 2008, p. 330), then it is reasonable to assume that the task of sharing it with patient or general public representations is no easier.

The point of introducing this issue is not to suggest that the exchange needs to be saddled with more information. However, it is a principle of pharmacoeconomics and the function of cost-utility analysis itself that the costs of medical interventions to health care systems are compared to their clinical, economic, and humanistic benefits (Bootman et al., 2005). Within the latter of these three categories there is an opportunity to introduce debates into healthrelated literature, especially as it connects to policy, about what constitutes humanistic benefits, to whom, how, and under what conditions. At each of these points the literature will be enhanced by input from humanities research. It is not even necessary to formally equip any of the participants with specific kinds of competencies about the broader contours of these kinds of debates, but to more simply stimulate a culture of learning and awareness about them. It should be comparable to the organic emergence of Medical Humanities, now part of the feedback loop that it helped to establish in the 1990s - but organized around policy decisions and the multiple variables that are brought to bear on them, of which health economics is one.

Unpacking categories like "humanistic" or "societal" might well involve deeper interrogation of symbolic, discursive, moral, and other qualitative issues. The most explicit recognition of qualitative evidence in policy decisions concerning the use of medical technologies has been advanced by Giacomini et al. (2001 \& 2010). The title of their most recent work (2010) indicates its full relevance to the present project: "Social Values and Health Technology Policy Analysis: The Role of Qualitative Social Science and Humanities Research Evidence." They cite the Ontario Health Technology Advisory Committee's (OHTAC) guidelines for determining decisions and isolate three of its features: relevant patient outcomes ("such as mortality, morbidity, quality of life"), societal values ("[b]roadly shared values which bear on the appropriate use and impact of the technology"), and ethical values, "[t]he potential ethical issues inherent in using or not using the technology" (p. 4). As a way to frame these 
dimensions and to facilitate an increase in their usage, Giacomini et al. (2010) use the thirtythree moral questions devised by Bjørn Hofmann for HTA decision-makers (2005). It is worth pausing over some of these questions. They are broken into five categories: moral issues, questions with respect to stakeholders, questions related to technology, moral aspects of methodological choices, and questions related to technology assessment. Four of these thirtythree questions are reproduced here, with Hofmann's original commentary included. They fall into the first two categories:

Q2. Does the implementation or use of the technology challenge patient autonomy? Many technologies can alter a person's self-determination. Drugs for sedation and surgical treatment of severely ill cancer patients are examples of where autonomy may be reduced.

Q5. Does the technology challenge human dignity? Technology for tracking patients with Alzheimer's disease is but one example.

Q8. Does the widespread use of a technology change our conception of certain persons (e.g., with certain diseases)? Technologies making it possible to screen for certain conditions, such as Down's Syndrome, may change esteem of persons with these conditions.

Q16. Can the technology harm the patient? New technology is highly potent for good and for bad. Explicitly addressing the potential harms is of moral import (p. 212-13).

Hofmann's moral questions are meant to clarify issues that are implicitly present in HTA. They emphasize the principles, values, and interests at stake, and the impact these issues have on social arrangements and institutions. Each of these topics yields meaningful contributions from the humanities, especially where autonomy, self-determination, and self-perception are concerned.

Giacomini and her co-authors acknowledge that deploying these kinds of inputs into the policymaking process is a logistical challenge, and they suggest some practical advice for doing so (Giacomini, 2004; Giacomini et al., 2001; Giacomini et al., 2010). Because qualitative and ethical reports are like "needles in haystacks," locating research that is relevant to the particular question or task at hand involves at a minimum searching databases beyond those usually associated with health technology. Steadfastness in this pursuit will often prove necessary since search filters for qualitative and ethical research are inadequate or nonexistent. Adding to this problem is the fact that qualitative reports and analyses are still rarely composed for the purpose of HTA, where interpretive and critical skills are necessary (Giacomini et al., 2010).

Practical attempts have been made to address some of these challenges and gaps in knowledge. Bombard et al. (2011) coordinated a 14-person participatory Citizens' Reference 
Panel as a contributor to the OHTAC's decision-making process. The methods used were varied and consisted of "deliberative discussions" and "pre- and post-questionnaires which assessed the relative importance of various ethical and social values as well as their stability over time" (Bombard et al., 2011, p.135). Bombard et al. (2011) conclude that a citizens' panel offers a promising way of eliciting qualitative values. They add that social values should be considered "at all stages of the health technology assessment process, and that relevant values should be considered on a case-by-case basis" (Bombard et al., 2011, p. 135, 143). There are training opportunities in HTA such as the Ulysses masters program and the Analyse et Évaluation des Interventions en Santé doctoral program that are open to applicants from humanities backgrounds (Hughes, 2009). Overall, serious attempts at incorporating moral issues into health care decision-making are increasing in number (Bombard et al., 2011).

Nevertheless, the relative paucity of attempts made at incorporating research that is explicitly derived from the humanities invites additional questions about the possibility of incongruent epistemological values, or at least differing disciplinary priorities. It is this last caveat described above by Bombard et al. (2011) - case-by-case knowledge, particularized rather than generalized, anchored in context - that has likely complicated the use of systematic humanities research enough that it has been marginalized in favour of quantitative research or ignored altogether. Although the principles of evidence-based medicine are beyond the scope of this essay, its hierarchy of evidence is a useful reminder of how data is typically prioritized by practitioners in this field. In the medical field, an emphasis on 'systematic reviews and randomized trials' is unsurprising given the importance of measurement and statistical rigour. But as Upshur et al. (2001) observe, although "values are regarded as central to health-care decision-making, the quantitative orientation to evidence proposed by advocates makes normative discourse seem an afterthought" (p. 91). This does not necessarily represent either a principled program of exclusion on the part of heath care researchers or policymakers or any attitude grounded in epistemological conviction concerning the purity of scientific data. It may simply be a reflection of the relatively recent emergence of the social, ethical, and moral dimensions of health-related policy decisions that might have formerly seemed settled without them. A recent retrospective on the history of medical humanities as a discipline indicates that its integrative nature has meant that "some have challenge[d] the academic credentials of this new enterprise because it cannot be clearly located within an established and respected parent discipline" (Evans and Greaves, 2010, p. 66). For others, "the whole point has been to create a new academic space capable of transcending conventional disciplinary rules" (Evans and Greaves, 2010, p. 66). This generalization is part epistemological and part logistical, and holds true for the role of humanities research in health-related policy decisions.

However, the responsibility of moving these learning processes forward does not fall entirely to clinical researchers, health economists, policy decision-makers, and patient representatives. Humanities scholars are, if anything, the party most responsible for the lack of visibility of humanities research, thanks to a broad professional habit of orienting their work along radical 
lines. Put briefly, since the 1960s the twin pressures of Marxist principles and post-war French philosophy have combined to assert a powerful influence on humanities departments in Europe and North America (Scruton, 1985). The effect has been a tendency to privilege the literature of protest, rejection, and transgression. This trend has been characterized by scholars of various political inclinations as a "culture of repudiation" (Scruton, 2007, p. 73) and a "culture of complaint" (Pocock, 2005, p. 9). These scholars argue that "political resistance," which is the primary end of such scholarship, consists of "using words in a subversive way, in academic publications of lofty obscurity and disdainful abstractness" (Nussbaum, 1999, p. 2). At its worst, the radical pose consists of a complete turn from the "material side of life" and all its accompanying ordinary "messy things" (Nussbaum, 1999, p. 2).

This failure is all the more egregious because humanities research is uniquely positioned to illuminate the symbolic, discursive, and contextualized features of health care issues for ordinary people. At its best, this research also opens the process to non-specialists, thirdparty participants, and stakeholders directly and indirectly involved in decisions by providing concepts and languages that might console them if they suffer and help them communicate their suffering in meaningful ways. In short, this research can enhance the quality of all the engagements described above by providing either the means for reflecting on the unique circumstances of medical issues or a framework for understanding them in various contexts.

Yet use of the phrase "ordinary people" might repel many humanities scholars accustomed as they are to occupying themselves with the radical, the subversive, and the subaltern. One recent entry in Medical Humanities exemplifies this orientation. Paul Ulhas Macneill (2011) expresses "concern about portrayals of the humanities and art as benign and servile in relation to medicine and health professions" (p. 85). Macneill's approach to this problem, which is highly germane to the present task only at the surface, is to examine examples of performance artists who submit their bodies to provocative modifications. Now further afield, Macneill's lofty purpose is nothing less than undermining "conceptions of the body, medicine, and humanity's relationship with technology" (Macneill, 2011, p. 85). This sort of would-be revolutionary social criticism, so typical since the 1960 s, is not likely to endear itself to the health professionals, policymakers, and other individuals who are faced with rather more ordinary while nevertheless urgent and difficult problems.

Although Macneill is not unique in this respect, it is necessary to point out that there are points of view that are directly contrary to his approach. Johanna Shapiro (2011) has forcefully made this case:

We realize, for example, that a patient's story is rarely 'just a story', but is rather the conscious and unconscious representation and performance of intricate personal motives and dominant meta-narrative influences ... Further, the academy's recent focus on transgressive, boundary-violating counter-narratives, while meant to right 
the balance of what constitutes acceptable, even valuable stories in medicine, may inadvertently trivialize more conventional, conformist stories as inauthentic. (p. 68)

Shapiro clearly indicates the stakes involved in prioritizing research interests in the manner typified by Macneill, but it may be stated in even stronger terms: humanities scholars have marginalized their own fields of study. Whereas health professionals, health economists, and policymakers progressively modify their methods to meet new challenges and attempt to negotiate their differences, many humanities scholars continue to wilfully squander - even consciously de-legitimize - the opportunities that such changing attitudes provide.

To conclude, there is a basis for introducing qualitative and humanities research into certain practices and processes of health-related policymaking. Formal categories - such as societal expectations and humanistic benefits - draw upon (but are not exhausted by) qualitative input, and there is consequently a wide scale of subject matter and analysis that may be meaningfully affixed to them. If knowledge translation aspires to authentic interdisciplinarity, it should not continue to pretend that colossal bodies of qualitative research into the human condition exist. This is a terrible failure of the imagination, and a failure to perceive the needs of individuals as fully as research, methodology, or practical resources allow. It is a credit to the participants of the policymaking processes identified here - health care professionals, clinical researches, health economists, policymakers, and patient advocates - that they are all, in their own way, committed to knowledge translation as a dynamic process informed by a myriad of diverse factors. There are opportunities to clarify and improve their efforts still. These opportunities range from simple and short-term, - such as research habits that are broadened by consulting professionals or databases beyond the conventionally quantitative, to the resource-dependent and long-term, - such as cultivating learning initiatives devoted to exploring the qualitative issues surrounding health care policy.

\section{Humanities Research and the Evidence of Experience}

The final section of this essay consists of two discrete examples of where humanities research and health care intersect, and explains how they can enlarge and improve the evidence brought to bear on certain kinds of policy decisions. The first is the nature of physical suffering, including how it is experienced and how the experience of suffering is communicated. The second is the narrativity of illness and how its form and content help illustrate the personalized experience of illness. These themes are introduced here where policy and policymaking are concerned because both relate to the complexities of communication that are particular to health-related states. These examples provide frameworks, metaphors, and vocabularies designed to ease the difficulties that arise when such communication must take place between occasionally divergent parties who nevertheless aim for negotiation and compromise. 
Policy decisions in health care require quantitative data supplied by clinicians and economists. This confluence of inputs make health care an especially complicated area for public policymakers. Health care involves an array of diagnostic and therapeutic options and uncertainties, patient preferences, values, and costs (Hunink \& Glaszious, 2003). Add to this the political and practical matters that policymakers must balance, and health care starts to resemble an awkwardly divergent web of individuals and motives (Wranik, 2008). To put it another way, the process is an art as well as a science:

It is an art in the primary sense that the making of policy involves the purposeful pursuit of ideas, beliefs, and goals that are valued by interests and citizens in a democracy but that are not easily or perhaps even properly, reducible to scientific study. (Doern, 1996, p. 15)

This is true even for health-related policy, which must rely heavily on quantitative evidence. The themes of pain and narrative, respectively, cannot be reduced to a scientific language, nor even to a language appropriate to the social sciences. They are no more, and certainly no less, than the qualitative investigations that produce evidence of experience. As such, they reflect and clarify personal experiences. If carefully analyzed, they will also be found to yield insight into the qualitative ideas, beliefs, and goals that underpin the art of policymaking including those constituting the humanistic, moral, and ethical dimensions of decision-making that were observed in the previous section.

Referring back to Hofmann's moral questions cited above, particularly Q2 and Q16, will help focus the following discussion of physical suffering. The first of these questions concerns an individual's autonomy, self-determination, and self-perception; the second concerns the potential for an intervention's harm. Translating answers to these questions requires, in the last instance, communicating information between any number of combined dynamics. It may involve communicating between, for example, a patient and a doctor in a clinical setting, or between the primary source and the researcher in an academic setting, or between and among clinicians and policy decision-makers in a HTA setting. However, before any of these transactions can be satisfactorily carried out, a much more rudimentary problem should be confronted: namely the inexpressibility of pain. An important source of information in this respect must be the patient, and if she is not individually consulted, for example through a cost-utility analyses that draws upon both individualized and aggregate preferences (Coons \& Kaplan, 2005), she, and her points of view, will be framed in helpful ways in humanities research.

Elaine Scarry's The Body in Pain has established why the patient may have trouble communicating the nature of her pain, and by extension its effect on her self-perception and 
judgments of harm. In the words of Scarry (1988) "when one speaks about 'one's own physical pain' and about 'another person's physical pain,' one might almost appear to be speaking about two wholly distinct orders of events" (p. 4). This is because "[w]hatever pain achieves, it achieves in part through its unsharability, and it ensures this unsharability through its resistance to language" (p. 4). Quoting Virginia Woolf, Scarry observes that the English language can express the thoughts of Hamlet and the tragedy of Lear, but "let a sufferer try to describe a pain in his head to a doctor and language at once runs dry" (p. 4). This passage is worth quoting at length:

True of the headache, Woolf's account is of course more radically true of the severe and prolonged pain that may accompany cancer or burns or phantom limb or stroke, as well as of the severe and prolonged pain that may occur unaccompanied by any nameable disease. Physical pain does not simply resist language but actively destroys it, bringing about an immediate reversion to a state anterior to language, to the sounds and cries a human being makes before language is learned. (p. 4)

Additionally, there is research that suggests individuals attach meaning to their pain, including symbolic meaning, which in turn may affect its perceived intensity or the person's willingness to submit to treatment or medication (Sofaer, 1998).

These variables underscore the problems associated with meaningful communication among the parties described above about issues with serious consequences. These communication problems arise not only for specific individuals but also for policymakers and their advisers when weighing costs and benefits. Where Q2 and Q16 are concerned, it is evident that their respective answers will not be straightforwardly affirmative or negative, but rather highly textured by qualitative values. If physical pain is, or is expected to be, a factor in the outcome of a health-related policy, both its experience and its communication will be confounded by not only the limitations of language, but also the symbolic or contextual meanings attached to it. Humanities research should take the lead in navigating this sort of terrain.

The second theme is the role of narrative in how illness is understood and communicated. Here Q5, Q8, and Q16 are pertinent. When these questions are asked by policymakers and their advisers, the answers may be surprising, and it is a credit to humanities research that these unexpected dimensions might be drawn out. Illness narratives are generated by the ill, used in varying ways by clinicians, and have very recently been studied in depth by literary critics. Shapiro (2011) and Arthur Frank (1997) have made significant contributions to this scholarship. Shapiro's idea of "narrative humility" consists, first, of critical awareness of potentially unreliable narratives and narrators. But second and more importantly, it requires that such awareness take place "within a compassionate and humble context," where stories "are not objects to be comprehended or mastered, but rather dynamic entities that we approach and engage with, while simultaneously remaining open to ambiguity and contradiction" (2011, p. 70-71). Ultimately, Shapiro (2011) concludes, 
The patient's story belongs to the patient, not to the physician and not to the literary scholar, and needs to be approached with humility, respect, and honouring, as well as mastery and critique. (p. 71)

Shapiro's use of 'we' might be extended from the physician and the literary scholar to the participants of the policymaking process. Where these participants are not conscientious consumers of such narratives, perhaps they ought to be, especially if they are involved in decisions that have implications for how individuals engage with their illnesses and the policy interventions that might affect them. As Shapiro notes, the dynamic quality of such narratives places demands on the reader (or listener), who will require a mastery of sorts, or at least some basic vocabulary, for understanding them. In this case, humanities research has not only clarified the place and quality of illness narratives, but may also be a valuable source of edification for those finding themselves tasked with thinking about them.

Frank's related concept of "remission society" brings the illness narrative and Hofmann's questions together succinctly. This hypothetic society consists of all those who have been treated by medical interventions but who could never be considered cured. It includes cancer survivors, the chronically ill, those in chronic pain as a result of their treatment, the disabled, and those living with prostheses or mechanical body regulators. These people return from illness, but "obligations are never again what used to be normal" (Frank, 1997, p. 8-11). "The ethical questions for members of the remission society," observes Frank, "are not adjudications of health care conflicts, but how to live a good life while being ill" (1997, p. 156). Since the good life is shaped by qualitative decisions such as the meaning of pain and is plotted in narratives of varied characteristics, the effects that health-related policy decisions will have on individuals will be difficult to measure. Ideas about human dignity, perception of self, others, and harm, raise difficult questions for health-related policymakers. These have also long been questions of capital importance in moral philosophy. Given their relevance, there should be a more significant commitment among all those involved in health-related policy research, formulation, and advising to consider them at greater length.

\section{Conclusion}

Policy studies itself opens a space for the compelling influence of ideas, defined as one of three broad analytic dimensions (along with actors and institutions) (Howlett, Ramesh, \& Perl, 1990, p. 13). With this basis as a constant reminder, along with the more recent efforts of Giacomini and others, it is hoped that Hofmann's questions will be asked, and that they will be pursued in light of the many-sided and qualitative insights provided by humanities research. This essay has followed Giacomini et al., Bombard et al., and Hofmann in stressing the place of qualitative research in HTA. However, the concerns expressed throughout this essay, as well as the frameworks suggested, may be adapted for other contexts where health and policy meet. It may be as simple as stimulating humanities scholarship devoted specifically to critically examining the intersection of these processes from a practical and qualitative point of 
view. It may also be as complicated and logistically difficult as bringing humanities research and professionals into decision-making as partners or advisers where, on a case-by-case basis, their familiarity with the evidence of experience and other themes might prove valuable. Such efforts can also facilitate the process of policy analysis and decision-making by providing a common language for framing these qualitative concerns. This is especially true when those concerns resist clear communication - as in the extent and meaning of suffering - or when they appear in narratives that exist in a variety of communicable forms. Knowledge translation theory adequately encompasses the complexities of quantitative data, but the space it accords the qualitative data described herein is still far too limited. 


\section{References}

Attaran, A. (2009). Take your medicine?: The risk of patient-led litigation in Canada's medical access system. McGill Journal of Law and Health, 3, 3-20.

Bombard, Y., Abelson, J., Simeonov, D., Francois-Pierre, G. (2011). Eliciting ethical and social values in health technology assessment: A participatory approach. Social Science and Medicine, 73, 135-144. http://dx.doi.org/10.1016/j.socscimed.2011.04.017

Bootman, J. L. (2005). Introduction to pharmacoeconomics. In J. L. Bootman, R. J. Townsend, \& W. F. McGhan (Eds.), Principles of pharmacoeconomics (pp. 1-18). Cincinnati, $\mathrm{OH}$ : Harvey Whitney Books Company.

Coons, S. J., Kaplan, M., R. (2005). Cost-utility analysis. In J. L. Bootman, R. J. Townsend \& W. F. McGhan (Eds.), Principles of pharmacoeconomics (pp. 117-147). Cincinnati, $\mathrm{OH}$ : Harvey Whitney Books Company.

Davis, D. (2006). Continuing education, guideline implementation, and the emerging transdisciplinary field of knowledge translation. The Journal of Continuing Education in the Health Professions, 26, 5-12.

Office of Technology Assessment. (1976). Development of medical technology: Opportunities for assessment. Retrieved from: http://www.fas.org/ota/reports/7617.pdf http://dx.doi.org/10.1002/chp.46

PMid:16557510

Doern, G. B. (1996). The evolution of Canadian policy studies as art, craft, and science. In L. Dobuzinskis, M. Howlett, \& D. Laycock (Eds.), Policy studies in Canada: The state of the art (pp. 15-26). Toronto, ON: University of Toronto Press.

Evans, H. M., Greaves, D.A. (2010). Ten years of medical humanities: A decade in the life of a journal and a discipline. Medical Humanities, 36, 66-68.

http://dx.doi.org/10.1136/jmh.2010.005603

PMid:21393283

Frank, A. W. (1997). The wounded storyteller: Body, illness, and ethics. Chicago, IL: University of Chicago Press. 
Giacomini, M., Hurley, J., Gold, I., Smith, P., Abelson, J. (2001). 'Values' in Canadian health policy analysis: What are we talking about? Retrieved from:

http://www.chsrf.ca/migrated/pdf/researchreports/ogc/giacomini final.pdf http://dx.doi.org/10.1258/1355819041403222

Giacomini, M. (2004). Interdisciplinarity in health services research: Dreams and nightmares, maladies and remedies. Journal of Health Services Research and Policy, 9 (3), 177183.

Giacomini, M., Abelson, J., Goldsmith, L., Levin, L., DeJean, D., \& Smith, A. (2010). Social values and health technology policy analysis: The role of qualitative social science and humanities research evidence. Proceedings from Canadian Agency for Drugs and Technologies in Health '10: Symposium. Halifax, NS.

Graham, I. D., Logan, J., Harrison, M. B., Strauss, S. E., Tetroe, J., Caswell, W., \& Robinson, N. (2006). Lost in knowledge translation: Time for a map?. The Journal of Continuing Education in the Health Professions, 26, 13-24. http://dx.doi.org/10.1002/chp.47 PMid:16557505

Harden, J. (2000). Language, discourse and chronotype: Applying literary theory to the narratives in health care. Journal of Advanced Nursing, 31 (3), 506-512. http://dx.doi.org/10.1046/j.1365-2648.2000.01303.x PMid:10718868

Ho, K., Chockalingham, A., Best, A., Walsh, G. (2003). Technology-enabled knowledge translation: Building a framework for collaboration. Canadian Medical Association, 168 (6), 710-11.

Hofmann, B. (2005). Towards a procedure for integrating moral issues in health technology assessment. International Journal of Technology Assessment in Health Care, 21 (3), 312-318. http://dx.doi.org/10.1017/S0266462305050415 PMid:16110710

Howlett, M., Ramesh, M., \& Perl, A. (1995). Studying public policy: Policy cycles \& policy subsystems. Oxford, UK: Oxford University Press. 
Hughes, D. (2009). Developing ethics skills in the field of health technology assessment. The Evolution of Health-System Management and Evaluation Practices, 6 (1), 1; 7-8.

Retrieved from: http://www.medsp.umontreal.ca/getos/pdf/infoen022009.pdf

Hunink, M., \& Glasziou, P. (2003). Decision making in health care: Integrating evidence and values, volume I. Cambridge, UK: Cambridge University Press.

Landry, R., Amara, N., \& Lamari, M. (2001). Utilization of social science research knowledge in Canada. Research Policy, 30 (2), 333-349. http://dx.doi.org/10.1016/S0048-7333(00)00081-0

Lavis, J. N. (2006). Research, public policymaking, and knowledge-translation processes: Canadian efforts to build bridges. The Journal of Continuing Education in the Health Professions, 26, 37-45.

http://dx.doi.org/10.1002/chp.49

PMid:16557509

Lehoux, P., Tailliez, S., Denis, J.L., Hivon, M. (2004). Redefining health technology assessment in Canada: Diversification of products and contextualization of findings. International Journal of Technology Assessment in Health Care, 20 (3), 325-336. http://dx.doi.org/10.1017/S026646230400114X

PMid:15446762

Macneill, P. U. (2011). The arts and medicine: A challenging relationship. Medical Humanities, 37, 85-90.

http://dx.doi.org/10.1136/medhum-2011-010044

PMid:22114348

Nussbaum, M. (1999, February). The professor of parody. The New Republic, 22. Retrieved from: http://www.akad.se/Nussbaum.pdf

Pan-Canadian Cancer Drug Review Process. (2011). Retrieved from http://www.pcodr.ca/idc/groups/pcodr/documents/pcodrdocument/pcodr-newsreleasejuly13.pdf

Pocock, J. G. A. (2005). The discovery of islands: Essays in British history. Cambridge UK: Cambridge University Press.

Scarry, E. (1988). The body in pain: The making and unmaking of the world. Oxford, UK: Oxford University Press. 
Scruton, R. (1985). Thinkers of the new left. London, UK: Continuum Intl. Publishing Grp.

Scruton, R. (2007). Culture counts: Faith and feeling in a world besieged. New York, NY: Encounter Books.

Shapiro, J. (2011). Illness narratives: Reliability, authenticity and empathetic witness. Medical Humanities, 37, 68-72. http://dx.doi.org/10.1136/jmh.2011.007328 PMid:21757469

Sofaer, B. (1998). Pain: Principles, practice and patients. Cheltenham, UK: Nelson Thomas.

Tetroe, J. (2007). Knowledge translation at the Canadian institutes of health research: A primer. Focus: A Publication of the National Centre for the Dissemination of Disability Research, 18, 1-18.

Upshur, R. E. G., VanDenKerhof, E. G., \& Goel, V. (2001). Meaning and Measurement: An inclusive model of evidence in health care. Journal of Evaluation in Clinical Practice, 7 (2), 91-96.

http://dx.doi.org/10.1046/j.1365-2753.2001.00279.x

PMid:11489034

Wranik, D. (2008). Using economic evidence as a support tool for policy decisions: Herculean or Sisyphean effort?. Expert Review of Pharmacoeconomics and Outcomes Research, 8 (4), 329-332.

http://dx.doi.org/10.1586/14737167.8.4.329

Zwarenstein, M. \& Reeves, S. (2006). Knowledge translation and interprofessional collaboration: Where the rubber of evidence-based care hits the road of teamwork. The Journal of Continuing Education in the Health Professions, 26, 46-54.

http://dx.doi.org/10.1002/chp.50

PMid:16557506 